\title{
Lack of Medial Prefrontal Cortex Activation Underlies the Immediate Extinction Deficit
}

\author{
Seok Chan Kim, ${ }^{1}$ Yong Sang Jo, ${ }^{1}$ Il Hwan Kim, ${ }^{2}$ Hyun Kim,,${ }^{2}$ and June-Seek Choi ${ }^{1}$ \\ ${ }^{1}$ Department of Psychology, Korea University, Seoul 136-701, Korea, and ${ }^{2}$ Department of Anatomy, Korea University College of Medicine, Seoul 136-705, \\ Korea
}

We conducted a series of experiments to investigate the neural basis of the immediate extinction deficit, the lack of extinction when the interval between fear memory acquisition and extinction is short. In experiment 1, rats were given extinction training composed of 15 conditioned stimuli (CSs) either $15 \mathrm{~min}$ (immediate extinction: I-EXT) or $24 \mathrm{~h}$ (delayed extinction: D-EXT) after five tone-shock pairings. In the retention test performed $48 \mathrm{~h}$ after conditioning, I-EXT group exhibited significantly higher freezing than D-EXT group. In experiment 2, functional activation in the medial prefrontal cortex (mPFC) was detected using $c$-fos immunoreactivity. The number of Fos-positive neurons in the MPFC was significantly lower in I-EXT group than in D-EXT group. In experiment 3, rats received immediate extinction with microstimulation of the infralimbic region (IL) of the $\mathrm{MPFC}$, either contingently paired or unpaired with the CS. In a subsequent retention test, the paired stimulation group exhibited decreased freezing relative to the unpaired stimulation group. Together, our results suggest that the immediate extinction deficit may be linked to the lack of neuronal activity in the IL.

\section{Introduction}

Due to its enduring nature, modification of traumatic fear memories requires lengthy behavioral therapy and pharmacological intervention. To improve therapeutic efficacy, it is essential to understand the neural basis of fear memory regulation. Pavlovian fear conditioning and extinction has proven to be a useful laboratory model for studying neural regulation of fear responses (LeDoux, 2000; Kim and Jung, 2006). In a typical experiment, subjects initially acquire a fear memory by paired presentations of a neutral conditioned stimulus (CS) and an aversive unconditioned stimulus (US). Fear memory is manifested in the form of a conditioned response (CR), which includes autonomic and behavioral reactions. In a subsequent extinction session, the CS is repeatedly presented without the US, resulting in a reduced rate of fear CRs (Pavlov, 1927). Extensive evidence suggests that this reduction is a consequence of the generation of a separate extinction memory rather than unlearning of the conditioned fear response (Bouton, 2004). Unlike the original fear memory, the extinction memory is easily attenuated by changes in temporal and spatial parameters. For example, the extinction memory can be masked by the fear memory if the extinction context changes or if the interval between extinction and test is long (Bouton and Bolles, 1979; Rescorla, 2004).

Manipulations of the interval between fear acquisition and extinction critically influence the strength of the extinction mem-

\footnotetext{
Received Aug. 22, 2009; accepted Nov. 12, 2009.

This work was supported by the National Research Foundation of Korea (NRF) grant funded by the Korea government (MEST) (NO. 2009-008145) and by a grant (2009K001284) from Brain Research Center of the 21st Century Frontier Research Program funded by the Ministry of Education, Science and Technology, the Republic of Korea.

Correspondence should be addressed to June-Seek Choi, Department of Psychology, Korea University, 5-1 Anam, Seongbuk, Seoul 136-701, Korea. E-mail: j-schoi@korea.ac.kr.

DOI:10.1523/JNEUROSCI.4145-09.2010

Copyright $\odot 2010$ the authors $\quad 0270-6474 / 10 / 300832-06 \$ 15.00 / 0$
}

ory. Recent studies have demonstrated that extinction training administered shortly after the acquisition session does not effectively induce long-term suppression of the fear response (Maren and Chang, 2006; Woods and Bouton, 2008; Chang and Maren, 2009) [but see Myers et al. (2006) and Schiller et al. (2008)]. Several possible causes for this "immediate extinction deficit" (IED) have been suggested. Maren and Chang (2006) proposed that elevated fear levels from the acquisition session may be responsible for IED by demonstrating that IED was attenuated when the level of fear during the acquisition session was minimized. Alternatively, Woods and Bouton (2008) proposed, on the basis of their appetitive learning data, that a minimal delay is required for the acquisition memory to be formed and accessible for the next stage of learning. A similar hypothesis was proposed using an associative learning model with multiple state transitions (Wagner and Brandon, 1989). However, none of these studies have examined the basis of IED at the neuronal level nor determined the brain circuits that underlie this phenomenon.

The medial prefrontal cortex (mPFC) has been implicated in the formation and expression of extinction memory. Rats with damage to the mPFC have difficulty recalling extinction (Morgan et al., 1993; Morgan and LeDoux, 1995; Quirk et al., 2000). In addition, the tone responses of infralimbic cortex (IL) neurons are potentiated during retrieval, and their activity correlates with extinction memory. Moreover, local stimulation of the IL facilitated extinction, confirming a causal relationship (Milad and Quirk, 2002). We therefore reasoned that investigating the pattern of mPFC activation at the time of extinction might provide insights into the underling mechanisms of IED. If mPFC is involved in IED, then immediate extinction should lead to under-activation of the $\mathrm{mPFC}$ and failure to generate the neuronal changes necessary for extinction memory. Finally, artificial activation of the mPFC should be respected to revive immediate extinction. 
A

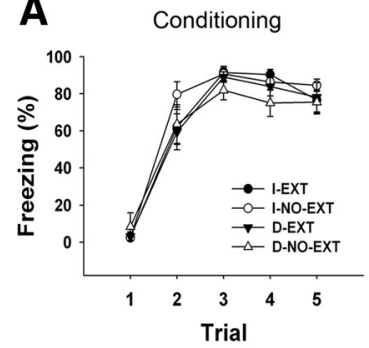

B
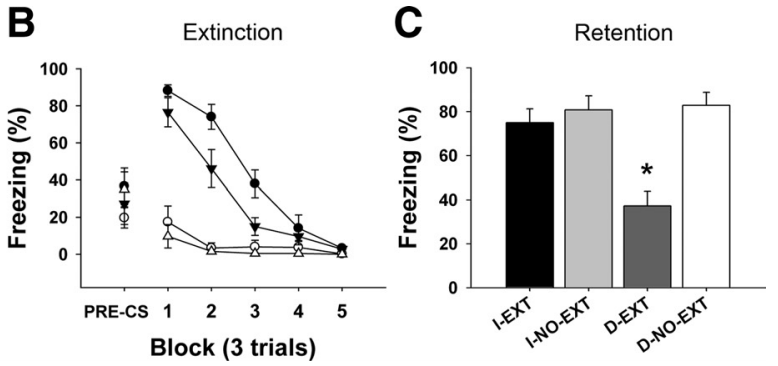

Figure 1. Immediate extinction deficit. A, Percentage of freezing during conditioning. Rats were given five CS-US conditioning trials. All groups exhibited a similar increase in freezing across trials. $\boldsymbol{B}$, Percentage of freezing during extinction training. The immediate extinction group (I-EXT) and the delayed extinction group (D-EXT) received 15 CS-only trials, 15 min and $24 \mathrm{~h}$ after fear conditioning, respectively. Both groups showed similarly attenuated freezing at the end of the extinction training. The immediate no-extinction group (I-NO-EXT) and the delayed no-extinction group (D-NO-EXT) were placed in the extinction context for the same amount of time but without $C S$ presentation at the corresponding time points. All tests were performed in a novel context. C, Averaged percentage of freezing across three test trials. The D-EXT group showed significantly lower freezing relative to the other groups. ${ }^{*} p<0.05$. All data are presented as means \pm SEM.

\section{Materials and Methods}

Experiment 1: behavioral data. Rats were subjected to three phases of training: fear conditioning, extinction, and extinction retention test. Rats received five presentations of the $\mathrm{CS}(2 \mathrm{kHz}, 30 \mathrm{~s}, 80 \mathrm{~dB})$ that coterminated with footshocks $(0.5 \mathrm{~s}, 1 \mathrm{~mA})$ in one context. Either $15 \mathrm{~min}$ (I-EXT, $n=13$ ) or $24 \mathrm{~h}$ (D-EXT, $n=11$ ) after conditioning, each group received extinction training in which 15 CSs were presented alone in a different context. Two control groups (I-NO-EXT, $n=12$ and D-NO-EXT, $n=$ 10) were exposed to the extinction context for the same duration of time in the absence of the CS at corresponding time points. All groups were subjected to three tone-alone trials in the extinction context $48 \mathrm{~h}$ after conditioning. Details of the fear conditioning procedures are described in the supplemental Methods (available at www.jneurosci.org as supplemental material).

Experiment 2: c-fos immunohistochemistry. Rats were subjected to behavior training procedures as described above, and then killed $90 \mathrm{~min}$ after the extinction training. Fos levels were evaluated in I-EXT $(n=4)$ and D-EXT groups $(n=3)$, as well as in four control groups: (1) immediate no extinction, (2) delayed no extinction, (3) immediate unpaired, and (4) delayed unpaired. The unpaired control groups (I-UNPAIRED, $n=3$ and D-UNPAIRED, $n=3$ ) received tones and shocks in an unpaired manner during the conditioning phase and were returned to the extinction context for 15 extinction trials at corresponding time points. The no-extinction groups (I-NO-EXT, $n=3$ and D-NO-EXT, $n=4$ ) were placed in the extinction context for the same amount of time but were not exposed to the CS at corresponding time points.

Fos levels were quantitatively analyzed in the IL, prelimbic cortex $(\mathrm{PL})$, and secondary motor cortex (M2). All procedures for examining Fos expression were similar to those previously described (Jo et al., 2007). Briefly, two images $(200 \times 200 \mu \mathrm{m})$ per brain region were selected for cell counting. All cell nuclei were identified by $4^{\prime}, 6^{\prime}$ diamidino-2-phenylindole (DAPI) staining (blue), and Fos expression was detected using a rabbit anti-c-fos antibody (1:1000; Calbiochem) and a cyanine 3 (CY3) goat anti-rabbit IgG (1:1000; Invitrogen) (red). A neuron with Fos expression in its nucleus was considered a Fosimmunoreactive (Fos-IR) cell. The number of Fos-IR cells in each image was counted blindly, and the ratio of Fos-IR neurons to DAPI-labeled cells per image was calculated.

Experiment 3: artificial mPFC activation. To stimulate neuronal activity of the IL, a bipolar stimulating electrode (Plastics One) was implanted in the IL $(0.125 \mathrm{~mm}$; impedance, $15-30 \mathrm{k} \Omega$ ) (see supplemental Methods, available at www.jneurosci.org). Rats were allowed to recover for $7 \mathrm{~d}$ and were then subjected to fear conditioning as described above. After conditioning, rats received electrical stimulation of the IL during immediate extinction training. The stimulation consisted of a $300 \mathrm{~ms}$ train of square pulses $(0.2 \mathrm{~ms}$ pulse width, $100 \mathrm{~Hz})$ produced by a pulse generator (MASTER-8, A.M.P.I), and was delivered through a stimulus isolator (A360R-C, World Precision Instruments) to maintain the current at 100 $\mu \mathrm{A}$. The paired-stimulation group (I-PAIRED; $n=21)$ received IL stimulation $100 \mathrm{~ms}$ after CS onset. In addition to I-PAIRED group, six control groups were included; unpairedstimulation groups (immediate or delayed), no-stimulation groups (immediate or delayed), stimulation-only group (immediate), and M2-stimulation group (immediate). The unpaired-stimulation groups randomly received explicitly unpaired tones and IL stimulation during the extinction training either 15 min (I-UNPAIRED; $n=16$ ) or $24 \mathrm{~h}$ (D-UNPAIRED; $n=11)$ after conditioning. The nostimulation groups (I-NO-STIM, $n=14$ and D-NO-STIM, $n=17$ ) were subjected to extinction training at the corresponding time points but did not receive IL stimulation, while the stimulation-only group (I-STIM; $n=9$ ) received IL stimulation without CS presentation during immediate extinction training. As an anatomical control, the M2-stimulation group (M2-STIM; $n=12$ ) received M2 stimulation paired with the CS during immediate extinction training.

\section{Results}

\section{Immediate extinction deficit}

To compare the effects of immediate and delayed extinction on long-term extinction memory without interference from baseline fear, we employed an $\mathrm{ABB}$ design using one context for acquisition and the other for extinction and extinction retention. All groups exhibited robust freezing at the last conditioning trial $\left(F_{(3,42)}=0.401, p=0.753\right)$ (Fig. $\left.1 A\right)$. For extinction training, all rats showed similarly low freezing during the $5 \mathrm{~min}$ baseline pe$\operatorname{riod}\left(F_{(3,42)}=0.750, p=0.529\right)$. At the beginning of extinction training, the I-EXT and D-EXT groups showed robust freezing which declined gradually, whereas the I-NO-EXT and D-NOEXT groups maintained low freezing throughout the session (Fig. $1 B)$. ANOVA revealed that there were main effects for group $\left(F_{(3,42)}=46.312, p<0.01\right)$ and trial block $\left(F_{(4,168)}=59.934, p<\right.$ $0.01)$, as well as a group $\times$ trial block interaction $\left(F_{(12,168)}=\right.$ 13.032, $p<0.01)$. Post hoc analysis confirmed that extinction groups froze significantly more than the no-extinction groups (all $p<0.01)$. In addition, the I-EXT group showed higher freezing than the D-EXT group $(p<0.01)$. A direct comparison between I-EXT and D-EXT groups revealed that the I-EXT group froze significantly more than the D-EXT group in the second and third trial block (all $p<0.05$ ). However, rats in both groups showed equally attenuated freezing by the end of the extinction training $(p=0.703)$, indicating that loss of freezing within the extinction session was equivalent in the two groups regardless of the postacquisition interval.

Despite an equivalent reduction in freezing during the extinction session, the two extinction groups differed in the retention test. ANOVA showed a main effect for group $\left(F_{(3,45)}=10.805\right.$, $p<0.01$ ), and post hoc analysis confirmed that the D-EXT group froze significantly less than the other groups (all $p<0.01$ ) (Fig. $1 C)$. However, the I-EXT group showed a high freezing level, which was similar to the no-extinction groups (all $p>0.05$ ).

It is possible that the higher level of freezing in the I-EXT group was due to slower extinction during the preceding session. To exclude this possibility, we matched the freezing levels between the two extinction groups ( I-EXT $=13, \mathrm{D}-\mathrm{EXT}=7$ ) by selecting a subset of rats (freezing level of $30 \%$ or greater during the second block of the extinction session). ANOVA revealed that there was no difference in freezing levels during extinction train- 


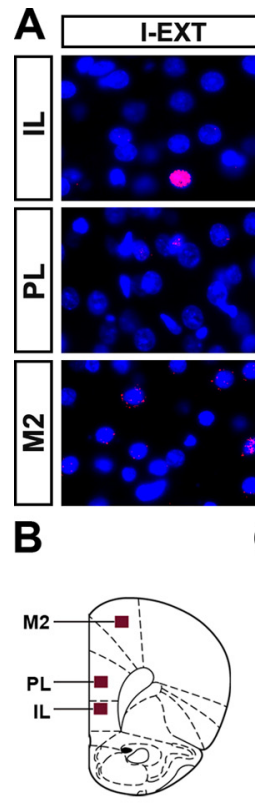

$3.20 \mathrm{~mm}$

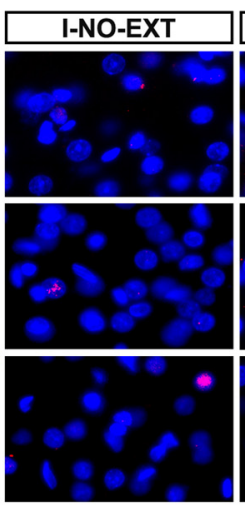

C

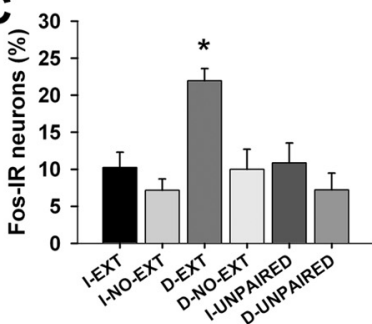

IL
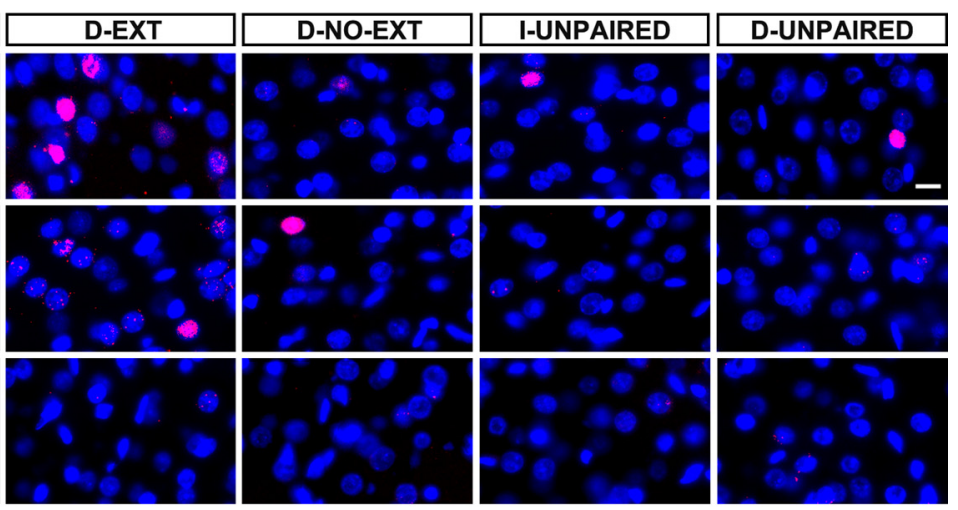

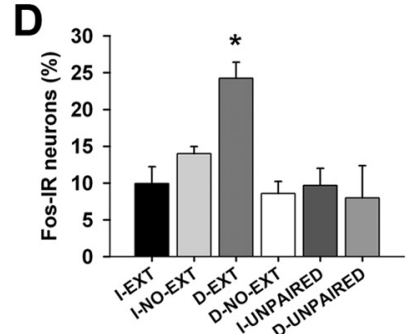

PL

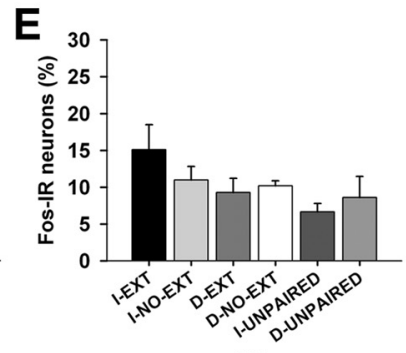

M2

Figure 2. Fos expression in the mPFC following immediate extinction. $\boldsymbol{A}$, Representative photomicrographs showing Fos expression in the IL, PL, and M2. Fos expression was identified using CY3 fluorescence (red), and nuclei were identified by DAPI staining (blue). Scale bar, $10 \mu \mathrm{m}$. B, Schematic diagram illustrating the IL, PL, and M2. C-E, Percentages of Fos-IR neurons in the IL, PL, and M2. Fos-IR neurons in both the IL and PL were found in significantly greater numbers in the D-EXT group than in other groups. Numbers of Fos-IR neurons in the I-EXT group were not different from the controls. D-EXT, Delayed extinction group; I-EXT, immediate extinction group; I-NO-EXT, immediate no extinction group; D-NO-EXT, delayed no extinction group; I-UNPAIRED, immediate unpaired conditioning group; D-UNPAIRED, delayed unpaired conditioning group. ${ }^{*} p<0.05$. All data are presented as means \pm SEM.

ing (group $\times$ trial block, $p=0.223$ ) (supplemental Fig. 1, available at www.jneurosci.org as supplemental material). In addition, the I-EXT group still showed a higher level of freezing than the D-EXT group $\left(t_{(18)}=3.277 ; p<0.01\right)$. Thus, our data indicate that extinction training conducted shortly after conditioning does not lead to long-term extinction memory, which is consistent with a previous report (Maren and Chang, 2006).

Fos expression in the mPFC following immediate extinction We next investigated the possibility that IED may be caused by a lack of neuronal activity in the mPFC. Behavioral training during the conditioning and extinction sessions was performed as described above (supplemental Fig. 2, available at www.jneurosci. org as supplemental material). Different levels of Fos expression in the IL and PL were observed among groups (Fig. $2 A$ ). ANOVA revealed a main effect of group on the number of Fos-IR neurons in the IL $\left(F_{(5,19)}=5.079, p<0.05\right)$ and the PL $\left(F_{(5,19)}=5.654\right.$, $p<0.05)$, but not in the M2 $\left(F_{(5,19)}=1.573, p=0.231\right)$ (Fig. $2 C-E)$. Post hoc analysis confirmed that the fraction of Fos-IR neurons in both the IL (all $p<0.05$ ) and PL (all $p<0.05$ ) was significantly greater in the D-EXT group than in the other groups. All the other groups, including the I-EXT group, displayed an equally low level of Fos expression in the IL (all $p>0.05$ ) and PL (all $p>0.05$ ) in comparison to D-EXT group. Therefore, our data suggest that IED results from a lack of neuronal activity in the mPFC.

The effects of IL stimulation during immediate extinction on long-term extinction memory

We next examined the possibility that IL stimulation during the immediate extinction could remedy IED. Only rats in which the electrode was precisely positioned in the IL were included in the analysis (Fig. 3B). All groups showed robust freezing at the last conditioning trial $\left(F_{(6,93)}=1.851, p>0.05\right)$ (Fig. 3C). During extinction training, all groups showed a similar pattern of reduction in freezing, except for the I-STIM group. ANOVA revealed that there were main effects for group $\left(F_{(6,93)}=3.076, p<0.01\right)$ and trial block $\left(F_{(4,372)}=64.585, p<0.01\right)$, as well as a group $\times$ trial block interaction $\left(F_{(24,372)}=2.499, p<0.01\right)$. Post hoc analysis confirmed that the I-EXT group froze significantly less than the other groups in the first trial block $(p<0.01)$ (Fig. 3D). These results indicate that electrical stimulation of the IL had no effect on the level of freezing during the extinction session. The retention test was conducted $48 \mathrm{~h}$ after fear acquisition in the absence of IL stimulation. A one-way ANOVA revealed a significant effect for group $\left(F_{(6,93)}=6.732, p<0.01\right)$. Post hoc analysis confirmed that the I-PAIRED group froze significantly less than other groups (all $p<0.05$ ), but did not differ from the D-NO-STIM $(p>0.05)$ or D-UNPAIRED groups $(p>0.05)$ (Fig. $3 E)$. Thus, our data suggest that IL stimulation paired with the CS mitigates IED.

\section{Discussion}

We conducted a series of experiments to investigate the neural mechanism that underlies the loss of long-term extinction memory following immediate extinction training (IED). We first confirmed that extinction training administered immediately after fear conditioning failed to induce long-term suppression of fear memory. Using $c$-fos immunoreactivity, we found that Fos expression in the $\mathrm{mPFC}$ was significantly lower in the immediate extinction group than in the delayed extinction group. Finally, we were able to mitigate IED by electrical stimulation of the IL paired with the presentation of the CS. Together, these findings provide compelling evidence that IL activation is necessary for the longterm suppression of fear CRs and that IED is caused by a lack of neuronal activity in the IL. 
A

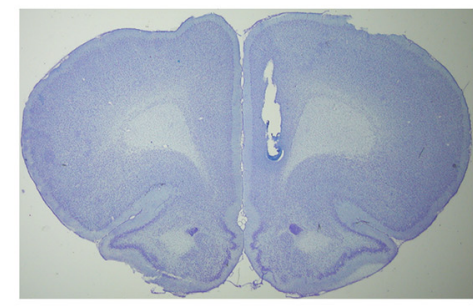

B

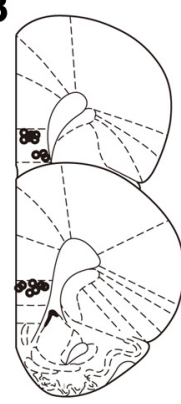

I-UNPAIRED

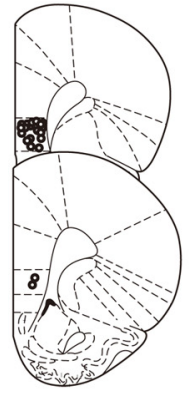

I-PAIRED

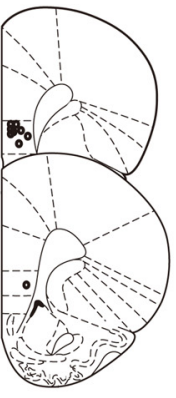

I-STIM

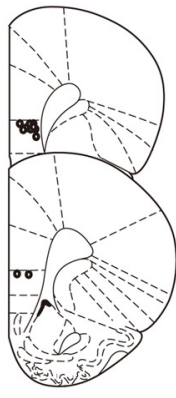

D-UNPAIRED

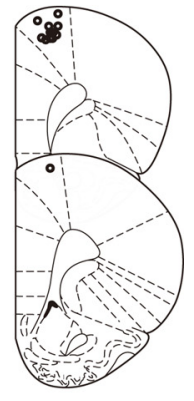

M2-STIM

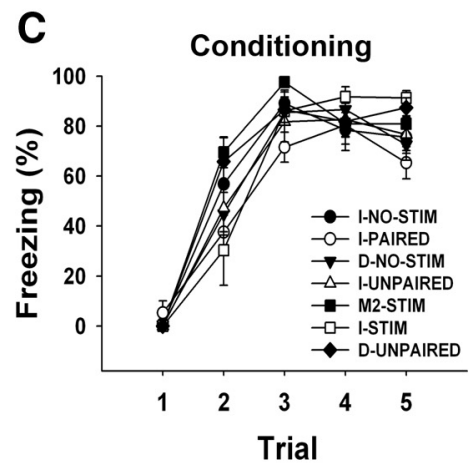

D
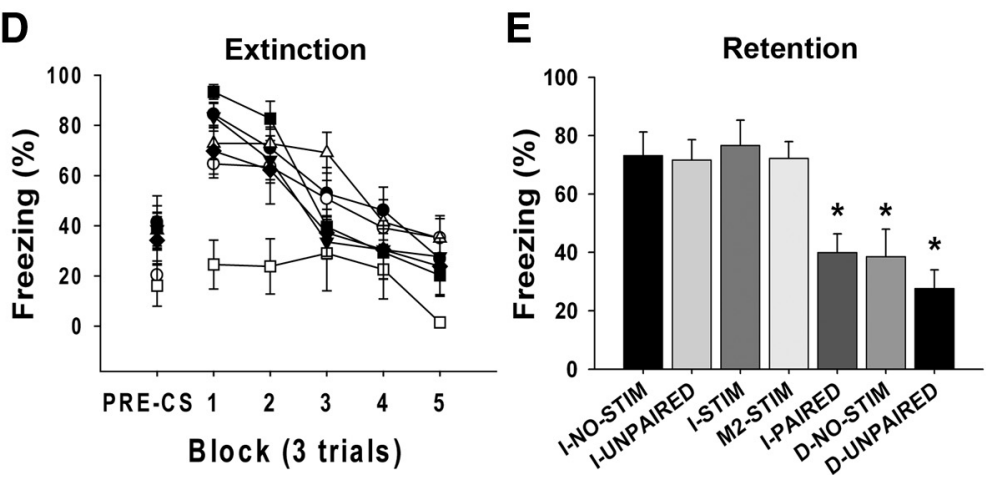

Figure 3. Effects of IL stimulation during immediate extinction training on long-term extinction memory. $\boldsymbol{A}, \mathrm{A}$ representative image showing a coronal section from a rat with a stimulating electrode in the IL. $\boldsymbol{B}$, Location of stimulation sites in the IL for the immediate unpaired-stimulation group, immediate paired-stimulation group, immediate stimulation-only group, delayed unpaired-stimulation group, and immediate M2-stimulation group [modified from Paxinos and Watson (1998)]. C, Percentage of freezing during conditioning. Rats were given five CS-US conditioning trials. All groups showed robust freezing in the last conditioning trial. D, Percentage of freezing during extinction training. The immediate paired-stimulation group (I-PAIRED) and M2-stimulation group (M2-STIM) received IL or M2 stimulation at $100 \mathrm{~ms}$ after CS onset during immediate extinction training, respectively. The immediate unpaired-stimulation group (I-UNPAIRED) and delayed unpaired-stimulation groups (D-UNPAIRED) received explicitly unpaired IL stimulation and tones at corresponding time points. The immediate no-stimulation and delayed nostimulation groups received extinction training at $15 \mathrm{~min}$ (I-NO-STIM) or $24 \mathrm{~h}$ (D-NO-STIM) after conditioning, but never received IL stimulation. In addition, the stimulation-only group (I-STIM) received IL stimulation in the absence of CS presentation. All groups showed a similar pattern of reduced freezing, except for the I-STIM group. $E$, Averaged percentage of freezing across three test trials. The I-PAIRED group froze significantly less than other groups, but did not differ from the D-NO-STIM or D-UNPAIRED groups. ${ }^{*} p<0.05$. All data are presented as means \pm SEM.

The results of the current study strongly support a role for mPFC activation in extinction, consistent with several previous [for review, see Myers and Davis (2002) and Sotres-Bayon et al. (2004)]. For example, extinction training induced $c$-fos immunoreactivity in the mPFC (Herry and Mons, 2004; Santini et al., 2004) and increased the excitability of IL neurons (Santini et al., 2008). In addition, IL stimulation paired with the CS facilitated extinction memory (Milad and Quirk, 2002; Milad et al., 2004). In the current experiment, delayed extinction also increased Fos expression in the mPFC. Following immediate extinction, however, few Fos-positive neurons were found, indicating that the $\mathrm{mPFC}$ was not fully active. The mPFC hypothesis was confirmed in the final experiment: artificial inflation of IL activity counteracted IED.

A key question that emerges from this study is why immediate extinction training was unable to activate the $\mathrm{mPFC}$. Considering the reciprocal projections and dynamic interaction between the amygdala and the mPFC (Cassell et al., 1989; McDonald, 1991; McDonald et al., 1996; Vertes, 2006), it is possible that the amygdala suppressed the $\mathrm{mPFC}$. This hypothesis is consistent with the observations that fear conditioning inhibited the excitability of IL neurons (Garcia et al., 1999; Ishikawa and Nakamura, 2003; Santini et al., 2008), and that stimulation of the basolateral amygdala decreased the responsiveness of mPFC neurons (PérezJaranay and Vives, 1991). In favor of this hypothesis, a high level of fear before the extinction session disrupted long-term extinction memory (Maren and Chang, 2006). However, in our study extinction training was performed in a different context to min- imize the initial fear level. As a result, little freezing was observed during the baseline period, indicating that the fear levels were low before the CS was presented. Thus, the loss of long-term extinction memory following immediate extinction training was not due to fear levels per se in our study.

Alternatively, inhibitory modulation of the MPFC might be mediated by stress response systems. Stress can be induced by intense physical stimulation or strong negative emotion (Selye and Horava, 1953). Once initiated, the effects of stress can linger on, even after the source of immediate stress is removed. Given that electric footshock elevated plasma levels of corticosterone for 90 min (Weinstock et al., 1998), stress levels may be higher in the immediate extinction condition than during delayed extinction, despite the lack of a measurable behavior (i.e., freezing) in both conditions. Activation of stress responses by the conditioning session could suppress the mPFC preventing it from becoming activated by extinction training. In support of this model, acute stress rapidly blocked the induction of long-term potentiation (LTP) in both amygdala-mPFC (Maroun and Richter-Levin, 2003) and hippocampus-mPFC (Rocher et al., 2004) pathways. Furthermore, acute stress induced dendritic retraction of pyramidal neurons in the IL, thereby causing an impairment in extinction (Izquierdo et al., 2006). From these results, it is reasonable to assume that the suppressed neuronal activity in the MPFC is the result of the temporally adjacent acquisition session, which involved physical and emotional stress.

Yet another hypothesis is that the associative learning memory must be encoded in the mPFC before a subsequent extinction 
memory can be processed, suggesting that a short postacquisition interval may not allow sufficient time for critical changes in the $\mathrm{mPFC}$ to occur. Retention of long- but not short-term memory in certain types of associative learning has indeed been shown to require delayed synaptic changes in the mPFC (TakeharaNishiuchi et al., 2006; Takehara-Nishiuchi and McNaughton, 2008). This hypothesis is also consistent with the role of the $\mathrm{mPFC}$ in other studies, which show that $\mathrm{mPFC}$ is not required for primary learning but becomes essential when experimental conditions, such as CS-US contingencies, are reversed in a subsequent phase (Schoenbaum et al., 2000). Similarly, one learning theory proposed a temporal memory model in which a "ready state" for extinction learning is not achieved until the acquisition phase has been stabilized (Wagner and Brandon, 1989). This "consolidation delay" hypothesis offers an explanation for the dynamic nature of extinction memory following appetitive as well as aversive learning (Woods and Bouton, 2008). Together, empirical data and theoretical work support that mPFC may play a role in prerequisite first stage learning, providing essential contribution to the second stage learning. However, further studies are needed to specifically test the time course of neural events in the mPFC following initial learning.

Although our data strongly support the theory that a short posttraining interval renders extinction training immensely ineffective, the optimal timing of early intervention following a traumatic event is still controversial. Some have reported that early intervention is effective in alleviating the severity of posttraumatic stress disorder (PTSD) symptoms (Campfield and Hills, 2001), whereas others have demonstrated that early intervention exacerbates trauma-related fear due to increased stress (Bisson et al., 1997; Gray and Litz, 2005). Results from laboratory experiments are equally confusing, perhaps due to differences in experimental protocols and parametric variations. For example, Myers et al. (2006) show that immediate extinction effectively eliminated spontaneous recovery, renewal, and reinstatement, implying the fear memory was permanently removed. On the other hand, Schiller et al. (2008) reported normal spontaneous recovery and reinstatement following immediate extinction in both humans and rats. It is difficult to directly compare these two studies as they differ in many significant ways, including the type of CR (potentiated startle vs freezing), US intensity, and the number of training trials. Further studies, especially those aimed at exploring different experimental parameters, are needed to elucidate which conditions differently drive the long-term consequences of immediate extinction. In the long run, this and related studies may inform development of optimal therapeutic options for mental disorders related to persistent fear memory.

\section{References}

Bisson JI, Jenkins PL, Alexander J, Bannister C (1997) Randomised controlled trial of psychological debriefing for victims of acute burn trauma. Br J Psychiatry 171:78-81.

Bouton ME (2004) Context and behavioral processes in extinction. Learn Mem 11:485-494.

Bouton ME, Bolles RC (1979) Role of conditioned contextual stimuli in reinstatement of extinguished fear. J Exp Psychol Anim Behav Process 5:368-378.

Campfield KM, Hills AM (2001) Effect of timing of critical incident stress debriefing (CISD) on posttraumatic symptoms. J Trauma Stress 14:327-340.

Cassell MD, Chittick CA, Siegel MA, Wright DJ (1989) Collateralization of the amygdaloid projections of the rat prelimbic and infralimbic cortices. J Comp Neurol 279:235-248.

Chang CH, Maren S (2009) Early extinction after fear conditioning yields a context-independent and short-term suppression of conditional freezing in rats. Learn Mem 16:62-68.

Garcia R, Vouimba RM, Baudry M, Thompson RF (1999) The amygdala modulates prefrontal cortex activity relative to conditioned fear. Nature 402:294-296.

Gray MJ, Litz BT (2005) Behavioral interventions for recent trauma: empirically informed practice guidelines. Behav Modif 29:189-215.

Herry C, Mons N (2004) Resistance to extinction is associated with impaired immediate early gene induction in medial prefrontal cortex and amygdala. Eur J Neurosci 20:781-790.

Ishikawa A, Nakamura S (2003) Convergence and interaction of hippocampal and amygdalar projections within the prefrontal cortex in the rat. J Neurosci 23:9987-9995.

Izquierdo A, Wellman CL, Holmes A (2006) Brief uncontrollable stress causes dendritic retraction in infralimbic cortex and resistance to fear extinction in mice. J Neurosci 26:5733-5738.

Jo YS, Park EH, Kim IH, Park SK, Kim H, Kim HT, Choi JS (2007) The medial prefrontal cortex is involved in spatial memory retrieval under partial-cue conditions. J Neurosci 27:13567-13578.

Kim JJ, Jung MW (2006) Neural circuits and mechanisms involved in Pavlovian fear conditioning: a critical review. Neurosci Biobehav Rev 30:188-202.

LeDoux JE (2000) Emotion circuits in the brain. Annu Rev Neurosci 23:155-184.

Maren S, Chang CH (2006) Recent fear is resistant to extinction. Proc Natl Acad Sci U S A 103:18020-18025.

Maroun M, Richter-Levin G (2003) Exposure to acute stress blocks the induction of long-term potentiation of the amygdala-prefrontal cortex pathway in vivo. J Neurosci 23:4406-4409.

McDonald AJ (1991) Organization of amygdaloid projections to the prefrontal cortex and associated striatum in the rat. Neuroscience 44:1-14.

McDonald AJ, Mascagni F, Guo L (1996) Projections of the medial and lateral prefrontal cortices to the amygdala: a Phaseolus vulgaris leucoagglutinin study in the rat. Neuroscience 71:55-75.

Milad MR, Quirk GJ (2002) Neurons in medial prefrontal cortex signal memory for fear extinction. Nature 420:70-74.

Milad MR, Vidal-Gonzalez I, Quirk GJ (2004) Electrical stimulation of medial prefrontal cortex reduces conditioned fear in a temporally specific manner. Behav Neurosci 118:389-394.

Morgan MA, LeDoux JE (1995) Differential contribution of dorsal and ventral medial prefrontal cortex to the acquisition and extinction of conditioned fear in rats. Behav Neurosci 109:681-688.

Morgan MA, Romanski LM, LeDoux JE (1993) Extinction of emotional learning: contribution of medial prefrontal cortex. Neurosci Lett 163:109-113.

Myers KM, Davis M (2002) Behavioral and neural analysis of extinction. Neuron 36:567-584.

Myers KM, Ressler KJ, Davis M (2006) Different mechanisms of fear extinction dependent on length of time since fear acquisition. Learn Mem 13:216-223.

Pavlov IP (1927) Conditioned reflexes: an investigation of the physiological activity of the cerebral cortex. London: Oxford UP.

Paxinos G, Watson C (1998) The rat brain in stereotaxic coordinates. San Diego: Academic.

Pérez-Jaranay JM, Vives F (1991) Electrophysiological study of the response of medial prefrontal cortex neurons to stimulation of the basolateral nucleus of the amygdala in the rat. Brain Res 564:97-101.

Quirk GJ, Russo GK, Barron JL, Lebron K (2000) The role of ventromedial prefrontal cortex in the recovery of extinguished fear. J Neurosci 20:6225-6231.

Rescorla RA (2004) Spontaneous recovery. Learn Mem 11:501-509.

Rocher C, Spedding M, Munoz C, Jay TM (2004) Acute stress-induced changes in hippocampal/prefrontal circuits in rats: effects of antidepressants. Cereb Cortex 14:224-229.

Santini E, Ge H, Ren K, Peña de Ortiz S, Quirk GJ (2004) Consolidation of fear extinction requires protein synthesis in the medial prefrontal cortex. J Neurosci 24:5704-5710.

Santini E, Quirk GJ, Porter JT (2008) Fear conditioning and extinction differentially modify the intrinsic excitability of infralimbic neurons. J Neurosci 28:4028-4036. 
Schiller D, Cain CK, Curley NG, Schwartz JS, Stern SA, Ledoux JE, Phelps EA (2008) Evidence for recovery of fear following immediate extinction in rats and humans. Learn Mem 15:394-402.

Schoenbaum G, Chiba AA, Gallagher M (2000) Changes in functional connectivity in orbitofrontal cortex and basolateral amygdala during learning and reversal training. J Neurosci 20:5179-5189.

Selye H, Horava A (1953) Stress research. Science 117:509.

Sotres-Bayon F, Bush DE, LeDoux JE (2004) Emotional perseveration: an update on prefrontal-amygdala interactions in fear extinction. Learn Mem 11:525-535.

Takehara-Nishiuchi K, McNaughton BL (2008) Spontaneous changes of neocortical code for associative memory during consolidation. Science 322:960-963.

Takehara-Nishiuchi K, Nakao K, Kawahara S, Matsuki N, Kirino Y (2006) Systems consolidation requires postlearning activation of NMDA recep- tors in the medial prefrontal cortex in trace eyeblink conditioning. J Neurosci 26:5049-5058.

Vertes RP (2006) Interactions among the medial prefrontal cortex, hippocampus and midline thalamus in emotional and cognitive processing in the rat. Neuroscience 142:1-20.

Wagner AR, Brandon SE (1989) Evolution of a structured connectionist model of Pavlovian conditioning (AESOP). In: Contemporary learning theories: Pavlovian conditioning and the status of traditional learning theory (Klein SB, Mowrer RR, eds), pp 149-189. Hillsdale, NJ: Erlbaum.

Weinstock M, Poltyrev T, Schorer-Apelbaum D, Men D, McCarty R (1998) Effect of prenatal stress on plasma corticosterone and catecholamines in response to footshock in rats. Physiol Behav 64:439-444.

Woods AM, Bouton ME (2008) Immediate extinction causes a less durable loss of performance than delayed extinction following either fear or appetitive conditioning. Learn Mem 15:909-920. 